\title{
Blood Pressure, Pulse, and Respiratory Changes Produced by Stimulation of the Hippocampus of the Monkey
}

\author{
Charles L. Votaw and Edward W. Lauer ${ }^{1}$ \\ Department of Anatomy, The University of Michigan, Ann Arbor, Michigan
}

Received February 4, 1963

Cardiovascular and respiratory changes resulting from systematic stimulation of the hippocampal formation of the monkey werc recorded. Stimulation of the anterior portion of the hippocampus resulted in a marked depression or cessation of respiratory excursions, but without apparent changes in respiratory rate, Respiratory changes were minimal to absent when posterior regions of the hippocampus were activated. Anterior hippocampal stimulation resulted in an initial depression of blood pressure accompanied by a small drop in pulse rate, followed by a rise in blood pressure which lasted throughout the period of stimulation and then gradually returned to former levels. The duration of the rise in blood pressure following cessation of stimulation was directly correlated with hippocampal activity. Posterior hippocampal stimulation did not show the secondary rise in pressure, only the depression. Marked pulse changes occurred only when the hippocampal gyrus was secondarily activated or stimulated directly. The neuron pathways which mediated these responses fall into two general groups, the fornix system and a hippocampotemporal system or both. A hypothesis that both systems may be concerned in visceral responses is presented.

\section{Introduction}

The fornix, in part, connects the hippocampus with the hypothalamus. Although some of its fibers are afferent with respect to the hippocampus (12), Brodal (3) has stressed the effectory structure of the hippocampus and the efferent nature of many of the fibers in the fornix. Though there is species variation in the distribution of fornix fibers, impulses traversing this pathway reach many of the nuclei of the hypothalamus, directly or indirectly $(21,22,23)$. The hypothalamus is an important internode

1 The authors gratefully acknowledge the support of this investigation by a University of Michigan Rackham Grant to C. L. V. and by National Science Foundation Grant G-11433. 
in the maintenance of proper autonomic regulation. The hippocampus, as the source of fornix fibers, may function to modify the physiological state of the viscera.

Papcz (26) suggested that the medial part of the telencephalon was concerned in the elaboration of visceral functions as well as the emotions. Fulton (10) and Thomalski, Klingler, and Woringer (35) discussed the limbic system as a controller of visceral functions. Others $(18,19,37)$ suggested that the hippocampus itself might have great influence on the vegetative processes. To investigate the latter possibility, cardiovascular and respiratory phenomena were recorded during stimulation of the monkey's hippocampus.

\section{Method}

One Macaca cynomolgus, one Cercopithecus aethiops and ten Macaca mulatta monkeys, without regard to sex, and weighing 2.3 to $4.7 \mathrm{~kg}$, were used. The animals were anesthetized with ether, intravenous sodium pentobarbital $(20 \mathrm{mg} / \mathrm{kg})$, or a combination of the two anesthetics. Stimulating electrodes consisted of insulated 24-gauge stainless steel tubing through which was passed an insulated silver wire, extending approximately 1.5 to $2 \mathrm{~mm}$ beyond the end of the tubing. Eight of these concentric electrodes, held in a stereotaxic carrier, were placed in the hippocampus at seven equal intervals from anterior to posterior, either by direct vision after removal of a small area of the overlying temporal lobe or according to previously determined stereotaxic coordinates, without first producing a cortical lesion. Placement of electrodes was later verified by study of histological sections. The stimulus consisted of square waves applied through a stimulus isolation unit. Current was measured by a cathode-ray oscilloscope. After preliminary trials, it was determined that best results were obtained at a stimulus intensity of $0.6-0.8 \mathrm{ma}$, a frequency of 40-60 per sec and a duration of 20-30 sec.

Results were recorded on a six-channel polygraph. Two channels were used to record the hippocampal electroencephalogram, one at the point being stimulated and the other at a more distant point. An electrocardiogram or a tachogram, which was triggered by the QRS wave of the electrocardiogram, was used to measure the heart rate. Blood pressure was recorded from a cannulated femoral artery by use of a suitable transducer. Respiration was measured by a pneumograph placed around the thorax of the monkey. A signal marker indicated the time of the stimulation. 
A series of stimulations werc made on each hippocampus of each monkey at different times. Repeat stimulations were not carried out until all parameters being measured had returned to prestimulation levels. Figure 1 illustrates the number of stimulations within each of the eight hippocampal areas arbitrarily established by the nature of the electrode holder.

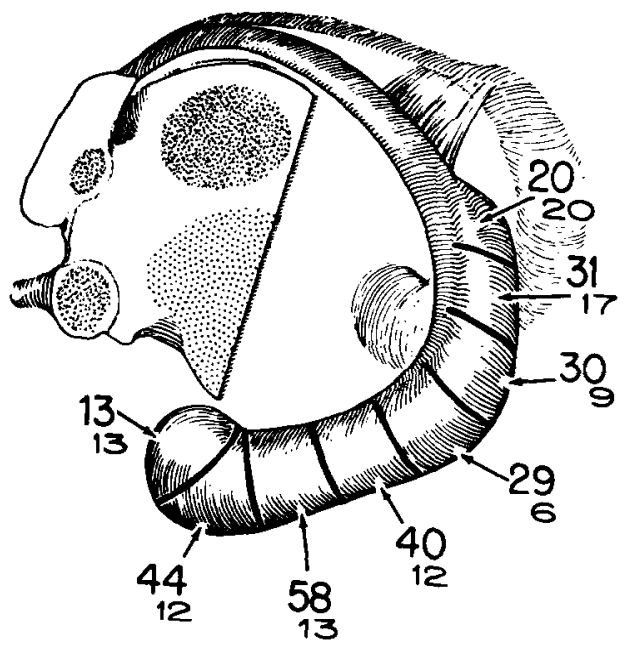

Fig. 1. Schematic drawing of the hippocampus of the monkey showing the eight divisions stimulated. Boldface numerals indicate the number of stimulations in each area when the hippocampus was directly exposed; the fineline numerals, the number by histologically proven, stereotaxically placed electrodes.

Aftcr these procedures, small lesions were produced by passing a direct current through the electrodes or by a high-frequency lesion maker. The animals were killed after a proper interval and the brains prepared by the Swank and Davenport modification of the Marchi technique (34).

\section{Results}

Respiration. Under the conditions of these expcriments the usual rate of respiration was found to range from 40 to 70 per $\mathrm{min}$. This figure agrees well with a mean value of 55 found by Gisler, Benson, and Young (11). Because of the nature of the measuring system used, no direct information was obtained on actual amount of tidal volume of air respired, but a rough estimate of increased or decreased respiratory volume was possible. 
Stimulation of the anterior half of the hippocampus, including the pes hippocampi, usually resulted in a marked depression of respiration. This most often appeared as a complete cessation of respiration in expiration (Fig. 2A). Stimulation of the most posterior regions of the hippocampus had no effect, or only a very transient one, as on the first inspiration after the onset of stimulation in Fig. 2B. There was a gradual lessening of the response as the stimulation was shifted more and more posteriorly.

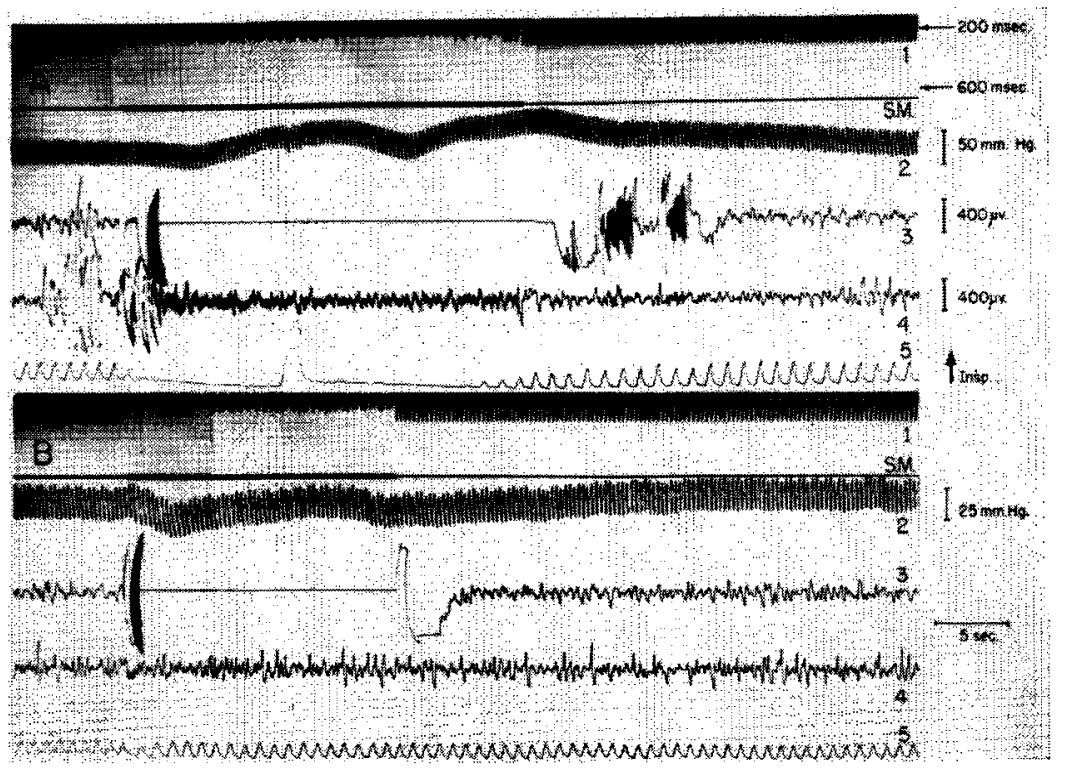

Fig. 2. Examples from the record resulting from stimulation of the anterior hippocampus (A) and of the extreme posterior hippocampus (B). Five channels are shown plus the signal marker between channels 1 and 2. Channel 1: tachogram, calibration expressed in msec between heart beats; SM.: signal marker, indicating the time of stimulation. Channel 2: blood pressure, baseline represents $100 \mathrm{~mm} \mathrm{Hg}$. Channel 3: hippocampal electroencephalogram at point stimulated. Channel 4: hippocampal electroencephalogram distant from the point stimulated. Channel 5: respiration (Insp., inspiration).

There was seldom any change at all in respiratory rate short of complete apnea. On occasion there was a deep, escape inspiration during the stimulation (Fig. 2A).

In about 50 percent of the instances where respiratory depression occurred, there was a deep inspiration immediately following stimulation (Fig. 3A). In other instances there was a gradual return over five 
or six respiratory efforts to a higher than prestimulation respiratory volume, followed, after a short time, by a return to the prestimulation norm (Fig. 2A). Occasionally this return of respiratory rhythm began before the stimulation was terminated.

The duration of the primary respiratory effect often lasted beyond the termination of stimulation (Fig. 3A). This period of decreased depth

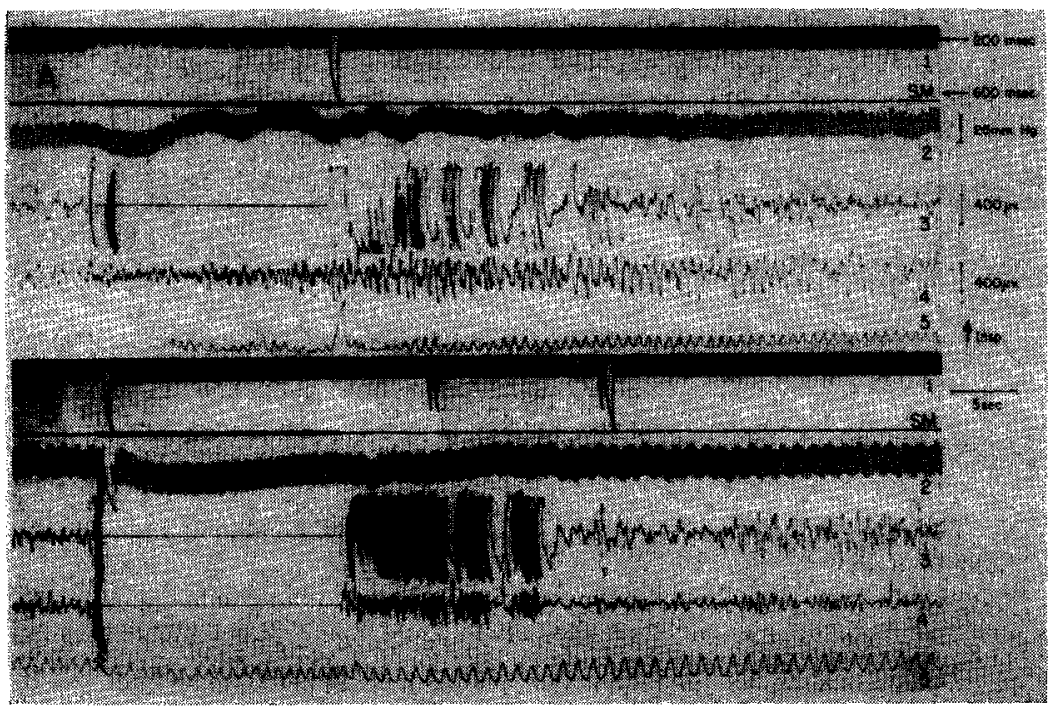

FIG. 3. Examples of records resulting from stimulation of the anterior hippocampus (A) and posterior hippocampus (B) from a different animal than that represented in Fig. 2. Arrangement of channels is the same as for Fig. 2.

of respiration was usually accompanied by afterdischarge or seizure activity in the hippocampus at the point stimulated. In most instances this increased activity did not spread to distant hippocampal areas. If stimulation extended beyond $30 \mathrm{sec}$, the animal either escaped from the primary inhibition or the response became irregular.

Stimulation of corresponding areas of two animals would not always give similar quantitative effects, but in any given animal stimulation anteriorly would produce an effect in the direction of apnea, and stimulation of the posterior area would give a much smaller to no effect (compare Figs. 2 and 3 ).

Blood Pressure. With the experimental procedures used, the systolic blood pressure varied from $110-150 \mathrm{~mm}$ of mercury at the beginning of 
each experiment. If the prestimulation level of blood pressure was ever lower than a systolic of 100 or if the interstimulation recovery level ever dropped more than $25 \mathrm{~mm}$ of $\mathrm{Hg}$ below the original value, the experiment was terminated. The pulse pressure varied from $20-35 \mathrm{~mm}$ of $\mathrm{Hg}$, a figure which is probably somewhat smaller than the actual value due to attenuation by the experimental technique. The true diastolic pressure is likewise higher than that shown in the records. In measuring the changes of blood pressure effected by stimulation, the systolic level was used.

Stimulation of the anterior third of the hippocampus generally caused an initial depression of $5-15 \mathrm{~mm}$ of $\mathrm{Hg}$. After 3-6 sec of stimulation, the blood pressure began to rise and reached a maximum of $20-35 \mathrm{~mm}$ of $\mathrm{Hg}$ above the prestimulation levels 10-15 sec after the onset of stimulation. The pressure remained high during the rest of stimulation, following which it returned gradually to the prestimulation level (Fig. 2A). If hyperactivity occurred in the hippocampus following stimulation, the blood pressure remained high until the termination of this hyperactivity (Fig. 3A). Within these general changes there were smaller variations which are independent of those produced by respiration. The changes seldom followed a smooth course.

The rise in blood pressure became smaller as more posterior points were stimulated, being very small or nonexistent at the caudal end of the hippocampus (Figs. 2B and 3B). The drop in blood pressure remained about the same, but the latency of maximum depression, in most instances, was prolonged (compare Fig. 3A and B). There was, however, a slow return to the previous level before stimulation ceased. There was occasionally a second depression during stimulation (Fig. 2B) and frequently after termination of stimulation, particularly if there was seizure activity in the hippocampus at the point stimulated (Fig. 3B).

Pulse. The range of sensitivity of the illustrated tachograms (Figs. $2,3,4$ ) was set for a 200-600 msec interpulse time. Stimulus artifacts are common but by comparing the tachogram with the pulse pressure the validity of a pulse rate change can be established. A slowing of pulse rate should normally result in an increase, and a quickening of the pulse in a decrease in pulse pressure. Because of the artifact, the pulse rate during stimulation was often determined from the blood pressure curve.

The prestimulation pulse rate varied from 190 to 250 beats per min. This is higher than a median pulse rate of 193 for the monkey reported 
by Gisler, Benson, and Voung (11). This slight difference is probably due to the experimental conditions.

For those stimulations in which we were reasonably certain there was no spread of the stimulation, there was a slight drop of 15-25 beats per min in the pulse rate occurring simultaneously with the fall of blood pressure. This appears small, but this change was in the opposite direction to that expected. If the normal reflex mechanism is operative, a fall of blood pressure should induce a rise in pulse rate. Following the fall, there was frequently a slight rise of $5-10$ beats per min in the pulse rate above the prestimulation level. This change was most prominent on stimulation of the anterior part, but did occur occasionally from activation of the posterior regions of the hippocampus.

On occasion, there developed, about midway during a 30-sec stimulation to the anterior portions of the hippocampus, a marked drop of 100-150 beats per min in the pulse rate, accompanied by a marked increase in the pulse pressure (Fig. 4A). This rate drop occurred during

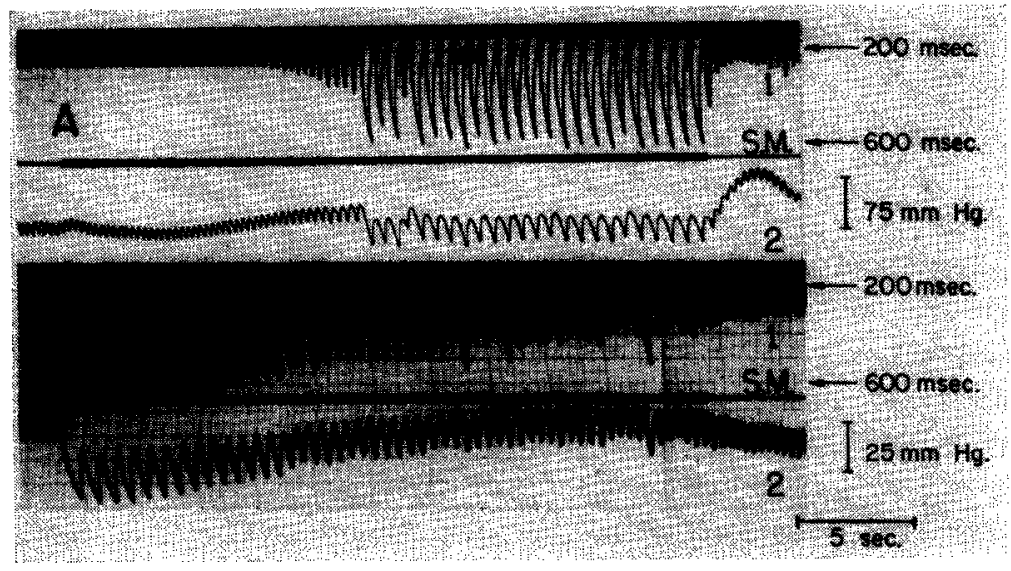

Fig. 4. Tachogram (1) and blood pressure (2) records from stimulation of the anterior hippocampus (A) and stimulation of the hippocampal gyrus (B) in the same anterior-posterior plane as A. S.M., signal marker.

a fall of blood pressure. Following stimulation, there was an abrupt return to normal pulse rate simultaneously with a rise of blood pressure. Figure 4B shows the tachogram and blood pressure curves resulting from stimulation of the midregion of the hippocampal gyrus of another animal in the same anterior stereotaxic plane as the result shown in Fig. 4A. 
The fall of pulse rate now occurred immediately upon stimulation, along with a fall of blood pressure and an increase in the pulse pressure. During stimulation there was a slow return toward prestimulation levels. It can be postulated that the radical change of pulse ratc which occurred late during stimulation of the hippocampus (Fig. 4A) was due to a spread of activity to the hippocampal gyrus.

Comparison with Stimulation of Topographically Close Structures. Figure 5 is from a record of a stereotaxic stimulation to the posterior region of the corticomedial amygdaloid complex in the same anterior

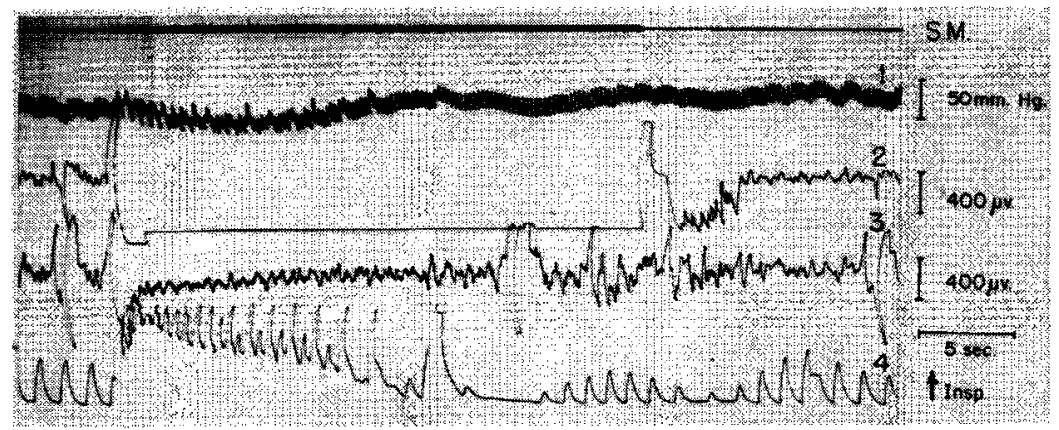

Fic. 5. A record from stimulation of the corticomedial amygdala. S.M., signal marker. Channel 1: blood pressure, baseline represents $100 \mathrm{~mm} \mathrm{Hg}$. Channel 2: electroencephalogram taken at the point stimulated. Channel 3: electroencephalogram distant from point stimulated. Channel 4: respiration (Insp., inspiration).

stereotaxic plane as the anterior portion of the hippocampus. Results of stimulation at this point differed from those obtained from the hippocamus. With the onset of stimulation, there was an increase in the inspiratory volume and respiratory rate, changes which were not seen with stimulation of the hippocampus. The pulse became irregular at a time when the blood pressure had lowered somewhat (stimulus artifact made the tachogram unusable in this record), a condition not observed when the hippocampus was stimulated. There was a slight fall in the blood pressure after the onset of stimulation but no rise above prestimulation levels as was observed from stimulation of the hippocampus at this same anterior plane.

These changes resulted from stimulation of an area of the brain very close to the hippocampus. This gives assurance that the results from hippocampal stimulation were not due to current spread. 


\section{Discussion}

The hippocampus is an effectory structure and the fornix is a prominent efferent pathway from it, leading, in part, to the hypothalamus (3). Since the hypothalamus is recognized as a center for autonomic regulation (1), it is somewhat surprising that Kaada (14) found no positive autonomic responses from stimulation of the hippocampus in primates, cat and dog, and that Penfield and Erickson (27) also found this area to be unresponsive, even in man. Pampligione and Falconer $(24,25)$ denied any autonomic responses from stimulation of the human hippocampus. Carlson, Gellhorn and Darrow (4) obtained autonomic changes from stimulation of the fornix within the hypothalamus in the cat but attributed these responses to current spread to hypothalamic nuclei.

Our experiments indicate that stimulation of the anterior portion of the hippocampus results in a decrease in respiratory effort. Anand and Dua (2) reported inconsistent findings on stimulation of the hippocampus of the monkey and the cat, but in at least one case, reported a temporary apnea. Segundo and his associates (32) reported a case of respiratory apnea in man secondary to stimulation of the fornix along the third ventricle. The apnea was in expiration and respiratory escapes occurred, findings similar to those reported here. Pool (31) also reported a single incidence of respiratory apnea in man from stimulation of the hippocampus.

Cragg (6) indicated an occasional apnea from stimulation of the fimbria of the rabbit, but, in this same reference and in Cragg and Hamlyn $(7,8)$, stimulation of the rabbit's dorsal fornix, the fimbria, and the ventral hippocampus produced an increase in rate and amplitude of respiration. It is possible that areas for both inhibition and facilitation of the respiratory process are found in the hippocampus. Our failure to note any type of facilitatory response would not support this conclusion.

Kaada (15), Kaada and Jasper (16), and Kaada, Pribram, and Epstein (17) have indicated that stimulation of the temporal tip, uncus, and anterior part of the hippocampal gyrus resulted in a respiratory arrest similar to our results. Poirier and Shulman (30) reported respiratory responses from much of the hippocampal gyrus and the ventral surface of the temporal lobe in the monkey. Because we stimulated a fairly intact system, as did the above investigators, the specific pathways involved are not known. It is possible that the response is mediated by 
way of the fornix which agrees with the experiments of Segundo and his associates (32). When Kaada (14) obtained respiratory responses from stimulation of the hippocampus in cat and monkey, he ascribed them as resulting from current spread to adjaccnt structures (c.g., caudatc nucleus). He also states here and elsewhere (15) that section of the fornix did not eliminate the respiratory response obtained from stimulation of the temporal cortex. Votaw (38) has shown the presence of hippocampo-temporal fibers going to those areas of the temporal lobe from which these respiratory responses were obtained. It is possible, then, that Segundo and his associates were stimulating hippocampal afferent fibers (12) and the discharge was through the temporal lobe. Perhaps Kaada (14) injured this discharge path and thus was unable to obtain respiratory changes from hippocampal stimulation.

Using cats and monkeys, Anand and Dua (2) reported a fall of blood pressure from stimulation of the hippocampus. Cragg and Hamlyn (6, 7, 8) reported a rise in blood pressure from stimulation of fimbria fibers which come mainly from the caudal third of the rabbit's hippocampus, and project to anterior hypothalamic regions, and a fall in blood pressure from stimulation of the fimbria fibers which arise from the anterior part of the hippocampus and project to the mamillary region. Comparatively, the anterior portions of the primate hippocampus are homologous to the caudal-most part of the rabbit's hippocampus and vice versa. Thus there is good correspondence between our results and those of Cragg and Hamlyn (ignoring for the moment the initial depression of blood pressure from stimulation of the anterior hippocampus). The true response is probably more complicated than just indicated.

Kaada and Pribram (17), Kaada (15), Wall and Davis (39), Poirier and Shulman (30) and Chapman, Livingston and Poppen (5) have all indicated that there are increases and decreases of blood pressure and pulse rate changes from stimulation of the anterior and anteroventral part of the temporal lobe. Marked pulse rate changes were only observed in our experiments when the hippocampal gyrus was activated. It is possible that cardiovascular functions of the hippocampus occur because of its connections with the temporal lobe.

The cardiovascular responses that we obtained and those reported in the references, point out a disassociation of the basic normal reflexes. Under physiological conditions, a rise of blood pressure would be accompanied by a reflex slowing of the pulse and vice versa, but we obtained a fall of blood pressure simultaneously with a fall of the pulse rate. This 
was most evident in stimulation of the hippocampal gyrus. It would appear that the effect of hippocampal stimulation on the cardiovascular system is not a simple inhibition or facilitation of basic reflexes, but a combination of a direct effect on heart rate and vascular tone, at the same time that certain basic reflexes are held inoperative. This might also account for the fluctuations which are noted in the responses, indicating a loss of the regular maintaining autonomic reflexes.

The pathways involved in these autonomic changes can only be surmised. Two general possibilities can be suggested. One is by way of the fornix projection system $(21,22,23)$. The other is from the hippocampus to the temporal lobe and then by various pathways to subcortical centers. Hippocampo-temporal fibers have been demonstrated by Votaw (38). Various investigators $(9,15,20,29,30,33,39)$ have indicated several pathways leading from the temporal lobe. There is also a close interrelation between the temporal cortical fields and the medial basal part of the frontal lobe, as has been shown by neuronography in the monkey by Petr, Holden and Jirout (28). Thus, another possibility is the connections from the temporal lobe to the frontal lobe from which visceral responses have been obtained $(13,17)$.

Torii and Kauamura (36) have noted in the cat that spontaneous increases of blood pressure were correlated with the appearance of hippocampal slow waves. The correlation of hippocampal electrical activity and blood pressure change was also seen upon stimulation of the amygdala. They concluded that hippocampal electrical activity may be an indicator of autonomic activity. We have noted that there is some correlation between afterdischarge, seizure activity of the hippocampus, or both, and the duration of the responses obtained following the end of stimulation. It is probable that activation of the hippocampus has a direct influence on the visceral regulations of the body.

\section{References}

1. Akert, K. 1960. Physiology and pathophysiology of the hypothalamus, pp. 152-229. In "Introduction to stereotaxis with an atlas of the human brain," vol. 1. G. Shaltenbrand and P. Bailey [eds.]. Georg Thieme Verlag, Stuttgart.

2. ANAND, B. K., and S. DuA. 1956. Circulatory and respiratory changes induced by electrical stimulation of limbic system (visceral brain). J. Neurophysiol. 19: $393-400$.

3. Brodal, A. 1947. The hippocampus and the sense of smell. Brain 70: 179-224.

4. Carlson, H. B., E. Gellhorn, and C. W. Darrow. 1941. Representation of the sympathetic and parasympathetic nervous systems in the forebrain of the cat. A.M.A. Arch. Neurol. Psychiat. 45: 105-116. 
5. Chapman, W. P., K. E. Livincston, and J. L. Poppen, 1950. Effects upon blood pressure of electrical stimulation of tips of temporal lobes in man. $J$. Neurophysiol. 13: 65-72.

6. CragG, B. G. 1958. Autonomic functions of the hippocampus. Nature 182: 675-676.

7. CragG, B. G., and L. H. Hamlyn. 1959. Histological connections and electrical and autonomic responses evoked by stimulation of the dorsal fornix in the rabbit. Exptl. Neurol. 1: 187-213.

8. CragG, B. G., and L. H. Hamiyn. 1960. Histological connections and visceral actions of components of the fimbria in the rabbit. Exptl. Neurol. 2: 581-597.

9. Crosry, E. C. 1956. The role of the midbrain as a part of the discharge paths from higher centers, pp. 217-231. In "Progress in Neurobiology." J. Ariëns Kappers [ed.]. Elsevier, Amsterdam.

10. Fulton, J.F. 1953. The limbic system: a study of the visceral brain in primates and man. Yale J. Biol. Med. 26: 107-118.

11. Gisler, D. B., R. E. Benson, and R. J. Younc. 1960. Colony husbandry of research monkeys. Ann. New York Acad. Sci. 85: 758-768.

12. Green, J. D. 1960. The hippocampus. Handbook Physiol, Secl. 1, Neurophysiol. 2: 1373-1389.

13. Ingram, W. R. 1960. Central autonomic mechanisms. Handbook Physiol., Sect. 1, Neurophysiol. 2: 951-978.

14. KAADA, B. R. 1951. Somato-motor, autonomic and electrocorticographic responses to electrical stimulation of rhinencephalic and other structures in primates, cat and dog. Acta Physiol. Scand. 24, Supp. 83: 1-285.

15. KaAda, B. R. 1960. Cingulate, posterior orbital, anterior insular, and temporal pole cortex. Handbook Physiol., Sect. 1, Neurophysiol. 2: 1345-1372.

16. KAADA, B. R., and H. H. JASPER. 1952. Respiratory responses to stimulation of temporal pole, insula and hippocampal and limbic gyri in man. A.M.A. Arch. Neurol. Psychiat. 68: 609-619.

17. Kaada, B. R., K. H. Pribram, and J. A. Epstein, 1949. Respiratory and vascular responses in monkeys from temporal pole, insula, orbital surface and cingulate gyrus. J. Neurophysiol. 12: 347-356.

18. KLÜvER, H. 1952. Brain mechanisms and behavior with special reference to the rhinencephalon. J.-Lancet 72: 567-574.

19. Maclean, P. D. 1949. Psychosomatic disease and the "visceral brain." Psychosomatic Med. 11: 338-353.

20. Marsan, C. A., J. Stolx, and H. H. Jasper. 1950. Electrophysiological studies of subcortical connections of the tip of the temporal iobe. Electroencephalog. Clin. Neurophysiol. 2: 356.

21. Nauta, W. J. H. 1956. An experimental study of the fornix system in the rat. J. Comp. Neurol. 104: 247-272.

22. NaUtA, W. J. H. 1958. Hippocampal projections and related neural pathways to the mid-brain in the cat. Brain 81: 319-340.

23. NaUta, W. J. H. 1959. A comparison of the distribution of the fornix system in the rat, guinea pig, cat, and monkey. J. Comp. Neurol. 113: 337-363. 
24. Pampiglione, G., and M. A. Falconer. 1956. Some observations upon stimulation of the hippocampus in man. Electroencephalog. Clin. Neurophysiol. 8: 718.

25. Pampiglione, G., and M. A. Falconer. 1960. Electrical stimulation of the hippocampus of man. Handbook Physiol., Sect. 1, Neurophysiol. 2: 1391-1394.

26. Papez, J. W. 1937. A proposed mechanism of emotion. A.M.A. Arch. Neurol. Psychiat. 38: 725-743.

27. Penfield, W., and T. C. Erickson. 1941. "Epilepsy and cerebral localization." Thomas, Springfield, Illinois.

28. Petr, R., L. B. Holden, and J. Jirout. 1949. The efferent intercortical connections of the superficial cortex of the temporal lobe (Macaca mulatta). J. Neuropath. Exptl. Neurol. 8: 100-103.

29. PoIRIER, L. J. 1952. Anatomical and experimental studies on the temporal pole of the macaque. J. Comp. Neurol. 96: 209-248.

30. PoIrIer, L. J., and E. Shulman. 1954. Anatomical basis for the influence of the temporal lobe on respiration and cardiovascular activity. J. Comp. Neurol. 100: 99-114.

31. Pool, J. L. 1954. The visceral brain of man. J. Neurosurg. 11: 45-63.

32. Segundo, J. P., R. Arana, E. Migliaro, J. E. Villar, A. Garcia Guelfi, and E. GaRCia AUSTT. 1955. Respiratory responses from fornix and wall of third ventricle in man. $J$. Neurophysiol. 18: 96-101.

33. Stoll, J., C. A. Marsan, and H. H. JASPer. 1951. Electrophysiological studies of subcortical connections of anterior temporal region in cat. J. Neurophysiol. 14: $305-316$.

34. Swank, R. L., and H. A. Davenport. 1935. Chlorate-osmic-formalin method for staining degenerating myelin. Stain Technol. 10: 87-90.

35. Thomalske, G., H. Klingler, and E. Woringer. 1958. Uber das Rhinencephalon. Acta Anat. 30: 865-901.

36. TORII, S., and H. KaUAMura. 1960. Effects of amygdaloid stimulation on blood pressure and electrical activity of the hippocampus. Japan. J. Physiol. 10: $374-384$.

37. Ule, G. 1954. Über das Ammonshorn. Forschr. Neurol. 22: 510-530.

38. Voraw, C. L. 1960. Certain functional and anatomical relations of the cornu Ammonis of the macaque monkey. J. Comp. Neurol. 114: 283-293.

39. WALl, P. D., and G. D. Davis. 1951. Three cerebral cortical systems affecting autonomic functions. J. Neurophysiol. 14: 507-517. 